\title{
The correlation of syndecan-4 with disease activity and serological characteristics of rheumatoid arthritis
}

Juan Zhao ( $\square$ juanzi810819@163.com )

Department of rheumatology and clinical immunology, Beijing, China

Xia Ye

Peking University First Hospital

Zhuoli Zhang

Peking University First Hospital

Research article

Keywords: rheumatoid arthritis, osteoarthritis, syndecan-4, sera, synovial fluid, disease activity

Posted Date: February 26th, 2021

DOI: https://doi.org/10.21203/rs.3.rs-248509/v1

License: (c) (i) This work is licensed under a Creative Commons Attribution 4.0 International License.

Read Full License 


\section{Abstract}

[Objectives]

To investigate the expression of syndecan-4 in serum, synovial fluid (SF) and synovium in rheumatoid arthritis (RA) by comparing with osteoarthritis (OA) patients, and to analyze the correlation of syndecan-4 with disease activity of RA .

[Methods]

Syndecan-4 in sera of 60 RA patients, 20 OA patients, 20 healthy controls, and in paired SF of 23 RA patients were tested by enzyme linked immunosorbant assay (ELISA). The expressions of syndecan-4 in synovium of 5 RA patients and 5 OA patients were detected by immunohistochemistry. The expressions of syndecan-4 of cultured synovial fibroblasts from RA and OA patients were detected by immunofluorescence. The correlation between serum syndecan-4 concentration and disease activity were analyzed in 60 RA patients.

[Results]

The serum syndedcan- 4 concentration was significantly higher in RA patients than in OA patients and healthy controls, and was higher in rheumatoid factor (RF)-positive RA patients than in RF-negative ones. Syndecan-4 concentration in SF of RA patients was comparable with OA patients. Syndecan-4 expression in synovial tissue was similar between RA and OA patients. The syndecan-4 concentration was significantly lower in synovial fluid than in serum, either in RA or in OA patients. The serum and SF syndecan-4 concentrations were both positively correlated with RA disease activity scores.

[Conclusion]

The serum syndecan-4 concentration is higher in RA patients than in OA patients, and significantly higher in RF-positive RA patients than in RF-negative ones. The serum and SF syndecan-4 concentrations were both positively correlated with disease activity of RA patients.

\section{Introduction}

Rheumatoid arthritis (RA), with morbidity of $0.36 \%$ in China, is a chronic systemic autoimmune disease characterized as articular synovitis and bone erosion that ultimately results in joint destruction. The aberrant B-cells activation, synovial fibroblasts proliferation and cartilage degradation are central features of RA. However, the pathogenesis of RA has not been clarified.

Syndecan-4, one of the members of transmembrane heparan sulphate proteoglycans (HSPGs), is the receptor of various cytokines and chemokines that can regulate a series of signal transductions [1]. It could be expressed in several cells under inflammation and involved in regulation of inflammation, angiogenesis, focal adhesion and cell migration [2]. Syndecan-4 ectodomains are constitutively shed and 
replaced under physiological conditions to maintain balance. However, in response to certain stimuli, such as extracellular cytokines, metalloprotease (MMP) and oxidative stress, syndecan-4 shedding increases dramatically, and the soluble syndecan-4 can promote inflammation and the synthesis of new syndecan-4 [3]. The cleavage and shedding of syndecans, which modulates the inflammatory response, was reported in lungs and heart $[4,5]$.

The role of syndecan-4 in inflammatory arthritis has been proved in animal models. Syndecan-4 deficient mice were resistant to the induction of collagen-induced arthritis (CIA) due to loss of the migration ability of B cells [6]. The tumour necrosis factor transgenic (hTNFtg) mouse model demonstrated that syndecan4 was prominently involved in synovial fibroblasts activation and its attachment to the cartilage in the early stage of arthritis [7]. Syndecan-4 deficient mice exhibited significantly reduced cartilage destruction compared with hTNFtg mice carrying syndecan-4 [7]. Intra-articular injection of anti-syndecan-4 antibodies into wild-type mice prevented cartilage damage to a similar extent as seen in syndecan-4deficient mice [8].

Several members of HSPG family, including syndecan-1, 2, 3, 4 and glypican-1, can be highly expressed by the fibroblast-like synoviocyte (FLS) of RA, but only syndecan-4 can interact with receptor protein tyrosine phosphatase sigma (RPTP $)$, a highly-expressed phosphatase in arthritic FLS which can regulate the RA FLS migration [9]. Syndecan-4 could up-regulate the levels of MMP with thrombospondin motifs (ADAMTS)-5 to promote the inflammation and cartilage degeneration [10]. A previous study reported little staining for syndecan-4 in synovium of early RA [12]. A most recent study demonstrated that highly expressed syndecan-4 was associated with decreased apoptosis in RA-FLS [11].

However, the levels of syndecan-4 in sera and synovial fluid (SF) of RA has not been reported. The expression of syndencan-4 in synovial tissue of RA is still controversial. The correlation of syndencan-4 with clinical features of RA, such as disease activity and the autoantibody, still remain unclear. The aim of the current study was to profile the expression of syndecan-4 in sera, SF and synovium in RA by comparing with OA patients and healthy controls, and analyze the correlation of serum syndecan-4 concentration with RA disease activity.

\section{Materials And Methods}

\section{Patients and samples}

The syndecan- 4 concentrations in sera were tested in 60 RA patients, and 20 OA patients and 20 healthy controls by enzyme linked immunosorbant assay (ELISA). The OA patients and healthy controls were matched with the RA patients by age and gender. The correlation of syndecan-4 concentration in sera and disease activity of RA patients were analyzed in the 60 RA patients.

23 RA patients had paired sera and SF samples. The sera and SF samples of one individual patient were collected at the same time point. Syndecan-4 concentrations in SF of the 23 RA patients and another 23 
OA patients were also tested by ELISA. The syndecan- 4 concentrations in SF of RA and OA patients were compared with the syndecan-4 concentrations in sera, respectively.

Expressions of syndecan-4 in synovial tissues of 5 RA patients and 5 OA patients were tested by immunohistochemistry. All the synovial tissues were derived from patients undergoing knee replacement. The RA patients and OA patients were also matched by age and gender.

All the above RA patients fulfilled the 2010 American College of Rheumatology/The European League Against Rheumatism (ACR/EULAR) classification criteria with various disease activity [from remission to high disease activity (HDA)]. All the enrolled OA patients fulfilled the OA classification criteria $[13,14]$. Patients with malignancies and other inflammatory diseases, including other inflammatory arthritis, connective tissue diseases and infections were excluded.

\section{Syndecan-4 concentrations in sera and synovial fluid}

Blood and SF were collected into tubes free of anticoagulants, centrifuged $3000 \mathrm{rpm}$ for $10 \mathrm{~min}$ to remove cellular debris and stored at $-80^{\circ} \mathrm{C}$ until use. Syndecan- 4 concentrations in sera and SF were measured by the human syndecan-4 ELISA kit (R\&D systems, the USA), following the manufacturer's protocols. Goat anti human syndecan-4 capture antibody was diluted to a terminal concentration of 0.8 $\mathrm{ug} / \mathrm{mL}$ using PBS, coated onto the wells of a polystyrene microtiter plate and incubated at room temperature overnight. Test sera without dilution were added to the microwells and incubated at room temperature for 2 hours. After washed for 3 times, a detective antibody with a terminal concentration of $100 \mathrm{ng} / \mathrm{mL}$ was then added and incubated at room temperature for 2 hours. Streptavidin-HRP was then added after washed for 3 times. After incubation for 20 minutes, substrate solution was added to the microwells. The reaction was terminated by the addition of stop solution and absorbance was measured at $450 \mathrm{~nm}$ and $540 \mathrm{~nm}$. Finally, sample concentration of syndecan-4 was determined by extrapolation in a standard curve prepared from seven geometric syndecan-4 standards.

\section{Immunohistochemistry}

The protocol of previous study was followed [15] with minor modifications by anti-syndecan-4 antibodies. Briefly, $10 \mathrm{~mm}$ thick serial cryostat sections of synovia were dried for 1 hour and stored at liquid nitrogen $\left(-196^{\circ} \mathrm{C}\right)$ until required. Prior to immunohistochemical analysis, slides were left to equilibrate to room temperature for 30 minutes, fixed in acetone $\left(4^{\circ} \mathrm{C}\right)$ for 10 minutes, air dried, and then sections were rehydrated in phosphate buffered saline (PBS) for 5 minutes. All primary antibodies were used at 5 $\mathrm{mg} / \mathrm{ml}$ since initial experiments showed that this concentration gave the optimum specific staining over the range of $2.5-10 \mathrm{mg} / \mathrm{ml}$. Antibody binding was detected using DAB staining kit (Vector Labs, Burlingame, California, USA). Sections were counterstained with Mayer Haematoxylin and mounted. In control experiments, isotype matched control mouse Ig or rabbit Ig were added instead of primary antibodies and stained with the DAB kit as above.

\section{Synovial fibroblast culture}


Synovial tissues were obtained from RA and OA patients during joint replacement surgery of knees. Tissues were minced and treated with $4 \mathrm{mg} / \mathrm{ml}$ type I collagenase (Gibco, USA) in serum free Dulbecco's modified Eagle's medium (DMEM; Gibco, USA) at $37^{\circ} \mathrm{C}$ for $1 \mathrm{~h}$. Primary cultured synovial cell lines were established and maintained in DMEM including $10 \%$ heat-inactivated fetal bovine serum (FBS; Gibco, USA), $1 \%$ penicillin-streptomycin ( $100 \mathrm{U} / \mathrm{ml}$; Gibco, USA), and 1\% L-glutamine ( $2 \mathrm{mM}$; Gibco, USA), in a humidified incubator at $37^{\circ} \mathrm{C}$ in the presence of $5 \% \mathrm{CO} 2$. Harvested cells were continuously cultured to obtain synovial fibroblasts, and cells of passages 3-6 were used in the next experiments.

\section{Immunofluorescence}

Cultured synovial fibroblasts of passages 3-6 were used for immunofluorescence. Cells were treated with rabbit anti-human syndecan- $4,4^{\circ} \mathrm{C}$ overnight. Washed with PBS and incubated with goat anti-rabbit IgGAlexa Fluor $\AA^{4} 88$ (Abcam, UK), room temperature for 1 hour. The biotinylated secondary antibody was detected by streptavidin-Alexa 594 (1:1000 Molecular Probes Code S-11227; Molecular Probes, Eugene, Oregon, USA).

\section{Statistical analysis}

All statistical analyses were performed using SPSS 20.0 (IBM Company, the USA) and all charts were performed by GraphPad Prism 5.0 (CA Company, the USA). The results of immunohistochemistry were analyzed by Image-Pro Plus 6.0 software (Media Cybernetics Company, the USA). Quantitative variables were expressed as mean \pm SD or median (min-max) for normal distribution or non normal distribution data, respectively. Statistical analysis was performed by the $\chi^{2}$ test (frequencies), nonparametric MannWhitney U test (non normal distribution data) and Student's $t$ test (normal distribution data), as appropriate. Correlation analysis was performed by Spearman correlation analysis. $P$ value less than 0.05 was considered significant.

\section{Results}

\section{Demographic and clinical features of RA patients}

60 RA patients as well as 20 age and gender matched OA patients and 20 healthy controls were enrolled in the study. Among 60 RA patietns, there were $12(20 \%)$ males and $48(80 \%)$ females with mean age of $54.8 \pm 11.6$ years and median disease duration of $6.3(0.5-34)$ years. $37(61.7 \%)$ of them were rheumatoid factor (RF) positive and 53 (88.3\%) were anti-cyclic citrullinated protein (anti-CCP) positive (Table 1). All the 60 patients were distributed in four different disease activity groups, including remission, low disease activity (LDA), moderate disease activity (MDA) and high disease activity (HDA), evaluated by disease activity score based on 28 joint count-C reactive protein (DAS28-CRP), DAS28-erythrocyte sedimentation rate (DAS28-ESR), simplified disease activity index (SDAI) and clinical disease activity index (CDAI).

The comparison of syndecan-4 in serum, synovial fluid and synovial tissue among RA patients, OA patients and healthy controls 
The syndecan-4 concentrations in sera of 60 RA patients, 20 OA patients and 20 healthy controls were $509.3(345.2-769.8) \mathrm{pg} / \mathrm{mL}, 363.6 \pm 137.6 \mathrm{pg} / \mathrm{mL}$ and $204.4 \pm 59.1 \mathrm{pg} / \mathrm{mL}$, respectively. The serum syndecan-4 concentration was significantly higher in RA patients than in OA patients and healthy controls $(p<0.001, p<0.001$, respectively). It was also higher in OA patients than in healthy controls $(p<0.001)$. (Figure 1)

In the 23 paired serum and SF samples of RA patients, the syndecan-4 concentrations was significantly lower in SF than in sera $(234.3 \pm 106.3 \mathrm{pg} / \mathrm{mL}$ vs $85.3 \pm 61.2 \mathrm{pg} / \mathrm{mL}, \mathrm{p}<0.001)$.(Figure $\mathrm{S} 1)$

The syndecan-4 concentrations was also significantly lower in SF than in sera of OA patients [363.6 $\pm 137.6 \mathrm{pg} / \mathrm{mL}$ vs $51.0(27.2-163.6) \mathrm{pg} / \mathrm{mL}, \mathrm{p}<0.001]$, although the sera and SF samples were not paired.

No significant difference of syndecan-4 concentrations in SF was found between the RA and OA patients $(p=0.733)$, although it was slightly higher in RA patients.

Syndecan-4 expression was detected by immunohistochemistry in the lining and sublining layers of all the 5 RA and 5 OA patents (Figure 2). The mean area, mean diameter, mean density and mean integrated optical density (IOD) were comparable between RA and OA patients (Table S1).

Immunofluorescence demonstrated that Syndecan-4 was positively stained in cultured synovial fibroblasts from both of RA and OA patients. The fluorescence intensity was similar between RA and OA patients. (Figure S2)

\section{The comparison of serum syndecan- 4 concentration between sera-positive and sera-negative RA patients}

The syndecan- 4 concentrations in sera were significantly higher in RF positive patients than in RF negative patients [586.2 (390.2-1124.8) pg/mL vs $434.3(312.0-537.5) \mathrm{pg} / \mathrm{mL},(\mathrm{p}=0.009)$ ]. The binary logistic regression showed that the OR was $1.003(1.000-1.005, p=0.022)$. For anti-CCP antibody positive and negative patients, it was comparable [517.4 (346.2-781.0) pg/mL vs 500.7 (373.8-537.5) pg/mL, $p=0.542]$ (Figure S3).

Then we defined patients with at least one of the above two antibodies positive as "sera positive", and there were 53 sera positive and 7 sera negative patients. The result was the same as that when stratified by anti-CCP antibody.

\section{The correlation between Syndecan-4 concentration with disease activity of RA patients}

Among the 60 RA patients, the syndecan-4 concentration in sera was positively correlated with TJC, SJC, PGA, EGA, and the disease activity scores, including DAS28-ESR, DAS28-CRP, SDAI and CDAI (Table 2). The correlation coefficents ( $r$ value) were mild to moderate (Table 2). Based on any of these disease activity scores, the patients in HDA had the highest serum syndecan-4 concentration, compared with those in remission, LDA or MDA (Table 3, Figure 3) 
Among the 23 RA patients, the syndecan-4 concentration in SF was positively correlated with PGA, EGA, CRP, DAS28-ESR, DAS28-CRP and SDAI (Table S2). All of the correlation coefficents ( $r$ value) were higher than 0.5 .

\section{Discussion}

In the current study, we found that syndecan-4 concentration in sera was positively related with the disease activity of RA. There were plenty of evidence proved the correlation between syndecan-4 and inflammation. The ectodomain of syndecan-4 could shed from the membrane under oxidative stress and inflammation $[17,18]$. Syndecan-4 can be up-regulated by TNFa, IL-1 $\beta$ and lipopolysaccharide (LPS) through a functional nuclear factor (NF)-кB site in its promoter [19]. TNFa can promote the shedding and decrease the attenuation of syndecan-4, both of which can amplify the effect of syndecan-4 [20]. Sheded heparan sulfate-substituted syndecan-4 ectodomains in turn increased ICAM-1, VCAM1, TNFa and IL-1 $\beta$ expression and NF-KB activation which all participate in the progression of RA, suggesting positive feedback regulation of inflammatory pathways [5].

Tenascin-C was considered essential in RA as high levels of its large-splice variant has been found in RA SF [21]. An immunodominant peptide from citrullinated tenascin-C was identified as a major target of autoantibodies in RA [22]. Tenascin-C might regulate the pannus invasion into cartilage. Syndecan-4 was required for tenascin- $C$ action. Inhibition of syndecan-4 suppressed tenascin-C activity and overexpression of syndecan-4 enhanced the effects of tenascin-C. Tenascin-C and syndecan-4 might work together to control fibroblast morphology and regulate matrix contraction [23]. This may one of the possible mechanisms by which Syndecan-4 participates in RA pathogenesis.

The exact pathways and mechanisms by which syndecan-4 contribute to inflammation and joint destruction in RA just began to emerge. A most recent published study demonstrated syndecan-4 silencing significantly suppressed the levels of reactive oxygen species (ROS), nitric oxide (NO) and inflammation and promoted the apoptosis of RA-FLSs. The apoptosis of RA-fibroblast-like synoviocytes (FLSs) regulated by syndecan-4 was mainly through the intrinsic pathway in which the P53, Caspase-3, B-cell leukemia/lymphoma 2 (Bcl2), and Bcl2-associated X protein (Bax) were involved. It provided evidence that Syndecan-4 mediated the inflammation and apoptosis of RA-FLSs by mediating the ROS and NO release [11].

We also found that the serum syndecan-4 level was significantly higher in RF positive RA patients than in RF negative ones. This might suggest that syndecan-4 participates in the activation of $B$ cells and the formation of autoantibodies of RA. Endo T et al. found that syndecan-4 deficient mice had reduced numbers and migration function of B cells, causing deficient germinal center (GC) formation in draining lymph nodes, suggesting that syndecan-4 may contributed to the development of CIA by promoting GC formation [6]. Unfortunately, we did not find difference between anti-CCP antibody positive and negative RA patients. This might be due to limited samples in the anti-CCP antibody negative group. 
B cells have been believed to play important roles in the pathogenesis of RA [24]. Syndecan-4 is one of the receptors of a proliferation inducing ligand (APRIL), which has been proved to be a critical signal for plasmablasts survival and an important molecular in the regulation of maturation and differentiation of $B$ cells [25]. It role of APRIL on the pathogenesis of RA had been revealed [26]. Our previous study also demonstrated that the soluble APRIL in sera was higher in RA than in OA patients, higher in sero-positive RA patients than in sero-negative ones, and more infiltration of APRIL positive cells in RA synovium than in OA patients [27]. The interaction between APRIL and syndecan-4 may contribute to the process of RA. Further study is needed.

Interestingly, we found the syndecan-4 concentration in sera was higher in OA patients than in healthy controls. Syndecan-4 levels in both of SF and synovium were similar in RA and OA, suggesting striking similarities in the pathogenesis of these two diseases. They are both degenerative joint diseases characterized by progressive loss of cartilage matrix and cartilage clefts. Increased MMP-3, MMP-13 and decreased bone morphogenetic protein-5 (BMP-5) have been found in both of RA and OA [28,29]. Syndecan-4 was closely related to cartilage destruction. The deficiency of syndecan- 4 in mice and intraarticular injections of syndecan-4 specific antibodies into wild-type mice can both protect from proteoglycan loss and thereby prevent OA cartilage damage [8]. Syndecan-4 regulates ADAMTS-5 activation which is crucial for the breakdown of cartilage matrix during $O A[8,10]$.

Syndecan-4 can be produced by a variety of different cells. In this study, it was observed that the concentration of syndecan-4 was significantly lower in SF than in sera either in RA or OA patients, suggesting that serum syndecan- 4 is not derived from syndecan- 4 produced in the joint. However, the syndecan-4 concentration in SF was also positively related with disease activity of RA. The correlation coefficents ( $r$ value) of syndecan-4 in SF with disease activity was even higher than that of syndecan-4 in sera with disease activity. It suggested that syndecan-4 played an important role in the inflammation of arthritis.

The correlations between syndecan- 4 and serological feature and disease activity of RA suggested syndecan-4 may participate in the pathogenesis of RA. It provided preliminary evidence to support syndecan-4 as a potential target for the prevention and treatment of RA.

\section{Conclusions}

The serum syndecan-4 concentration is higher in RA patients than OA patients and healthy controls, and significantly higher in RF-positive RA patients than in RF-negative ones. Serum and SF syndecan-4 concentrations are both positively correlated with the disease activity of RA patients. It may suggest that syndecan-4 may participate in the pathogenesis of RA and provided a novel therapeutic target for RA.

\section{Declarations}

- Ethics approval and consent to participate 
The study was approved by the Peking University First Hospital Biomedical Research Ethics Committee. The ICF obtained from study participants was written formally.

- Consent for publication

The submission was agreed by all the authors

- Competing interests

None.

- Funding

The study was supported by Peking University Clinical Research Program (PUCRP201305) and National Natural Science Foundation of China (No. 81771740, 81971524, 81901642).

- Authors' contributions

Dr Juan Zhao and Dr Xia Ye performed the laboratory test and analyzed the related data, Dr Juan Zhao collected and analyzed the clinical data and wrote the paper. Dr Zhuoli Zhang was the co-designer of the research and took the responsibility of the paper.

\section{References}

1. Kaneider, N. C., Feistritzer, C., Gritti, D., Mosheimer, B. A., Ricevuti, G., Patsch, J. R., et al. Expression and function of syndecan-4 in human platelets. Thrombosis and Haemostasis, 2005;93:1120-1127

2. Leonova El, Galzitskaya OV. Role of Syndecans in Lipid Metabolism and Human Diseases. Adv Exp Med Biol. 2015;855: 241-58.

3. Strand ME, Herum KM, Rana ZA, Skrbic B, Askevold ET, Dahl CP, et al. Innate immune signaling induces expression and shedding of the heparan sulfate proteoglycan syndecan- 4 in cardiac fibroblasts and myocytes, affecting inflammation in the pressure-overloaded heart. FEBS J. 2013;280:2228-47.

4. Xie J, Wang J, Li R, Dai Q, Yong Y, Zong B, et al. Syndecan-4 over-expression preserves cardiac function in a rat model of myocardial infarction. J Mol Cell Cardiol. 2012;53: 250-8.

5. Strand ME, Aronsen JM, Braathen B, Sjaastad I, Kvaløy H, Tønnessen T, et al. Shedding of syndecan4 promotes immune cell recruitment and mitigates cardiac dysfunction after lipopolysaccharide challenge in mice. J Mol Cell Cardiol. 2015;88:133-44.

6. Endo T, Ito K, Morimoto J, Kanayama M, Ota D, Ikesue M, et al. Syndecan 4 Regulation of the Development of Autoimmune Arthritis in Mice by Modulating B Cell Migration and Germinal Center Formation. Arthritis Rheumatol.2015;67:2512-22.

7. Korb-Pap A, Stratis A, Mühlenberg K, Niederreiter B, Hayer S, Echtermeyer F, et al. Early structural changes in cartilage and bone are required for the attachment and invasion of inflamed synovial 
tissue during destructive inflammatory arthritis. Ann Rheum Dis.2012;71: 1004-11.

8. Echtermeyer F, Bertrand J, Dreier R, Meinecke I, Neugebauer K, Fuerst M, et al. Syndecan-4 regulates ADAMTS-5 activation and cartilage breakdown in osteoarthritis. Nat. Med.2009;15: 1072-6.

9. Doody K M, Stanford S M, Sacchetti C, Svensson MN, Coles CH, Mitakidis N, et al. Targeting phosphatase-dependent proteoglycan switch for rheumatoid arthritis therapy. Sci Transl Med. 2015;288: 288ra76.

10. Wang J, Markova D, Anderson DG, Zheng Z, Shapiro IM, Risbud MV. TNF-a and IL-1 $\beta$ promote a disintegrin- like and metalloprotease with thrombospondin type I motif-5- mediated aggrecan degradation through syndecan-4 in intervertebral disc. J Biol Chem.2011;286:39738-49.

11. Cai P, Lu Z, Jiang T, Wang Z, Yang Y, Zheng L, et al. Syndecan-4 involves in the pathogenesis of rheumatoid arthritis by regulating the inflammatory response and apoptosis of fibroblast-like synoviocytes. J Cell Physiol. 2020;235:1746-1758.

12. Patterson A M, Cartwright A, David G, Fitzgerald O, Bresnihan B, Ashton BA, et al. Differential expression of syndecans and glypicans in chronically inflamed synovium. Ann Rheum Dis. 2008;67: 592-601.

13. Altman R, Asch E, Bloch D, Bole G, Borenstein D, Brandt K, et al. Development of criteria for the classification and reporting of osteoarthritis. Classification of osteoarthritis of the knee. Diagnostic and Therapeutic Criteria Committee of the American Rheumatism Association. Arthritis Rheum. 1986; 29:1039-49.

14. Altman R, Alarcón G, Appelrouth D, Bloch D, Borenstein D, Brandt K, et al. The American College of Rheumatology criteria for the classification and reporting of osteoarthritis of the hand. Arthritis Rheum. 1990;33:1601-10.

15. Roskams T, De Vos R, David G, Van Damme B, Desmet V. Heparan sulphate proteoglycan expression in human primary liver tumours. J Pathol 1998;185:290-7.

16. Jaap Fransen, Piet L C M van Riel. The Disease Activity Score and the EULAR Response Criteria. Rheum Dis Clin North Am. 2009;35:745-57.

17. Kliment CR, Englert JM, Gochuico BR, Yu G, Kaminski N, Rosas I, et al. Oxidative stress alters syndecan-1 distribution in lungs with pulmonary fibrosis. J Biol Chem. 2009; 284: 3537-45.

18. Kliment CR, Oury TD. Extracellular superoxide dismutase protects cardiovascular syndecan-1 from oxidative shedding. Free Radic Biol Med. 2011;50: 1075-80.

19. ME Strand, KM Herum, ZA Rana, B Skrbic, ET Askevold, CP Dahl, et al. Innate immune signaling induces expression and shedding of the heparan sulfate proteoglycan syndecan-4 in cardiac fibroblasts and myocytes, affecting inflammation in the pressure- overloaded heart, FEBS J. 2013;280: 2228-47.

20. Zhang Y, Pasparakis M, Kollias G, Simons M. Myocyte-dependent regulation of endothelial cell syndecan-4 expression. J Biol Chem.1999;274:14786-90.

21. Hasegawa M, Nakoshi Y, Muraki M, Sudo A, Kinoshita N, Yoshida T, et al. Expression of large tenascin-C splice variants in synovial fluid of patients with rheumatoid arthritis. J. Orthop. 
Res.2007;5: 563-8.

22. Schwenzer A, Jiang X, Mikuls TR, Payne JB, Sayles HR, Quirke AM. Identification of an immunodominant peptide from citrullinated tenascin-C as a major target for autoantibodies in rheumatoid arthritis. Ann Rheum Dis. 2016;75: 1876-83.

23. Midwood KS, Valenick LV, Hsia HC, Schwarzbauer JE. Coregulation of fibronectin signaling and matrix contraction by tenascin-C and syndecan-4.Mol Biol Cell. 2004;15:5670-7.

24. Kuhn KA, Kulik L, Tomooka B, Braschler KJ, Arend WP, Robinson WH, et al. Antibodies against citrullinated proteins enhance tissue injury in experimental autoimmune arthritis. J Clin Invest.2006;116:961-73.

25. Dillon SR, Gross JA, Ansell SM, Novak AJ. An APRIL to remember: novel TNF ligands as therapeutic targets. Nat Rev Drug Discov. 2006;5:235-46.

26. Nagatani K, Itoh K, Nakajima K, Kuroki H, Katsuragawa Y, Mochizuki M『et al. Rheumatoid arthritis fibroblast-like synoviocytes express BCMA and are stimulated by APRIL. Arthritis Rheum. 2007;56:3554-63.

27. Juan Zhao, Juan Guo, Lifang Wang, Wei Zhou, Zhuoli Zhang. (2014)The role of a proliferation inducing ligand (APRIL) in the pathogenesis of Rheumatoid Arthritis.Scand J Rheumatol. 2014;43:462-469.

28. Rose B.J., Kooyman D.L. A Tale of Two Joints: The Role of Matrix Metalloproteases in Cartilage Biology. Dis. Markers. 2016:4895050.

29. Bramlage CP, Häupl T, Kaps C, Ungethüm U, Krenn V, Pruss A, et al. Decrease in expression of bone morphogenetic proteins 4 and 5 insynovial tissue of patients with osteoarthritis and rheumatoid arthritis. Arthritis Res Ther. 2006;8:R58.

\section{Tables}

Table 1. The demographic and clinic features before and after 12 weeks treatment of TNFi+MTX in the cohort of 60 RA patients. 


\begin{tabular}{lc}
\hline & All RA patients $(\mathrm{n}=60)$ \\
\hline Age (mean \pm SD) & $58(47.3-63)$ \\
Female (\%) & $48(80.0)$ \\
Disease duration (years) (median, min-max) & $6.3(2.0-12.8)$ \\
RF positive (\%) & $37(61.7)$ \\
Anti-CCP antibody positive (\%) & $53(88.3)$ \\
TJC (median, min-max) & $3(1-10)$ \\
SJC (median, min-max) & $1(0-3)$ \\
PGA (0-100 mm) (median, min-max) & $40.0(20.0-60.0)$ \\
EGA (0-100 mm) (median, min-max) & $40.0(20.0-60.0)$ \\
HAQ-DI (0-3) (median, min-max) & $0.38(0.11-1.04)$ \\
ESR (mm/h) (median, min-max) & $18.5(9-39)$ \\
CRP (mg/L) (median, min-max) & $5.99(2.44-15.23)$ \\
DAS28-ESR (mean \pm SD) & $3.6(2.6-5.4)$ \\
DAS28-CRP (mean $\pm S D)$ & $3.2(2.6-5.1)$ \\
SDAI (median, min-max) & $11.9(6.6-29.2)$ \\
CDAI (median, min-max) & $11.5(6-27)$ \\
\end{tabular}

Note: RA: rheumatoid arthritis; RF: rheumatoid factor; anti-CCP: anti-cyclic citrullinated protein; TJC: tender joint count; SJC: swollen joint count; PGA: patient global assessment; EGA: evaluator global assessment; HAQ-DI: Health Assessment Questionnaire-Disability Index; ESR: erythrocyte sedimentation rate; CRP: C-reactive protein; DAS: disease activity score; SDAI: simple disease activity index; CDAI: clinical disease activity index.

Table 2. The correlation of serum syndecn-4 concentration with disease activity in 60 RA patients.

\begin{tabular}{lcc}
\hline & $\mathrm{r}$ & $\mathrm{p}$ \\
\hline TJC & 0.367 & 0.004 \\
SJC & 0.302 & 0.019 \\
PGA $(0-100 \mathrm{~mm})$ & 0.405 & 0.001 \\
EGA (0-100 mm) & 0.381 & 0.003 \\
HAQ-DI (0-3) & 0.131 & 0.320 \\
ESR (mm/h) & 0.128 & 0.331 \\
CRP (mg/L) & 0.109 & 0.408 \\
DAS28-ESR & 0.329 & 0.010 \\
DAS28-CRP & 0.356 & 0.005 \\
SDAI & 0.406 & 0.001 \\
CDAI & 0.413 & 0.001 \\
\hline
\end{tabular}

Note: RA: rheumatoid arthritis; TJC: tender joint count; SJC: swollen joint count; PGA: patient global assessment; EGA: evaluator global assessment; HAQ-DI: Health Assessment Questionnaire-Disability Index; ESR: erythrocyte sedimentation rate; CRP: C-reactive protein; DAS: disease activity score; SDAI: simple disease activity index; CDAI: clinical disease activity index. 
Table 3. The comparison of serum syndecn-4 concentration among different disease activity group in 60 RA patients.

\begin{tabular}{|c|c|c|c|c|c|}
\hline $\begin{array}{l}\text { syndecan- } \\
4 \\
(\mathrm{ng} / \mathrm{ml})\end{array}$ & Remission & $\begin{array}{l}\text { Low disease } \\
\text { activity }\end{array}$ & $\begin{array}{l}\text { Media disease } \\
\text { activity }\end{array}$ & $\begin{array}{l}\text { High disease } \\
\text { activity }\end{array}$ & $\mathrm{p}$ \\
\hline DAS28- & 432.2(287.4- & 519.2(177.1- & 484.8(343.6- & 680.1(466.3- & 0.082 \\
\hline ESR & 738.5) $(n=16)$ & 519.2) $(\mathrm{n}=3)$ & 677.8) $(n=25)$ & 1241.5) $(n=16)$ & \\
\hline DAS28- & 499.2(203.3- & 406.3(320.3- & 490.8(373.8- & 704.4(517.4- & 0.049 \\
\hline CRP & $537.5)(n=15)$ & 762.9) $(n=15)$ & 708.3) $(n=15)$ & 1263.3) $(\mathrm{n}=15)$ & \\
\hline SDAI & $\begin{array}{l}203.3(164.8- \\
203.3)(n=3)\end{array}$ & $\begin{array}{l}464.2(316.2- \\
743.4)(n=25)\end{array}$ & $\begin{array}{l}434.3(352.4- \\
501.0)(n=13)\end{array}$ & $\begin{array}{c}\text { 704.4(517.4- } \\
\text { 1176.2) }(\mathrm{n}=19)\end{array}$ & 0.014 \\
\hline CDAI & $\begin{array}{l}203.3(164.8- \\
203.3)(n=3)\end{array}$ & $\begin{array}{c}464.2(316.2- \\
743.4)(n=25)\end{array}$ & $\begin{array}{l}434.3(352.4- \\
501.0)(n=13)\end{array}$ & $\begin{array}{c}\text { 704.4(517.4- } \\
1176.2)(\mathrm{n}=19)\end{array}$ & 0.014 \\
\hline
\end{tabular}

Note: RA: rheumatoid arthritis; ESR: erythrocyte sedimentation rate; CRP: C-reactive protein; DAS: disease activity score; SDAI: simple disease activity index; CDAI: clinical disease activity index.

\section{Figures}

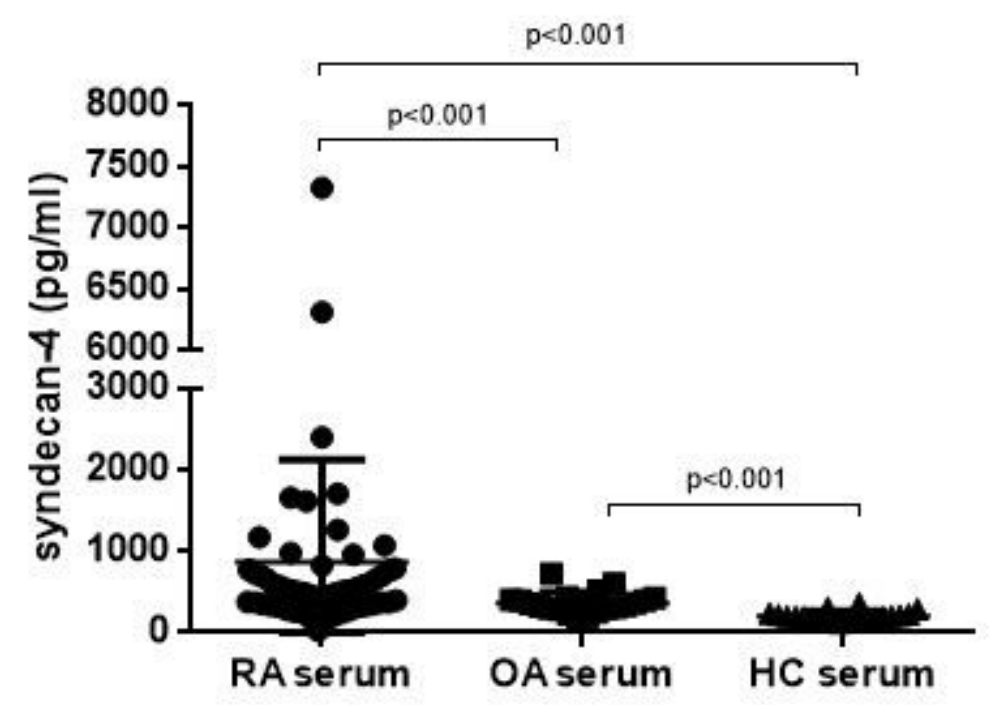

Figure 1

The comparison of Syndencan-4 concentrations among serum from 60 RA patients, 20 OA patients and 20 healthy controls. RA: rheumatoid arthritis; OA: osteoarthritis; HC: healthy control. 

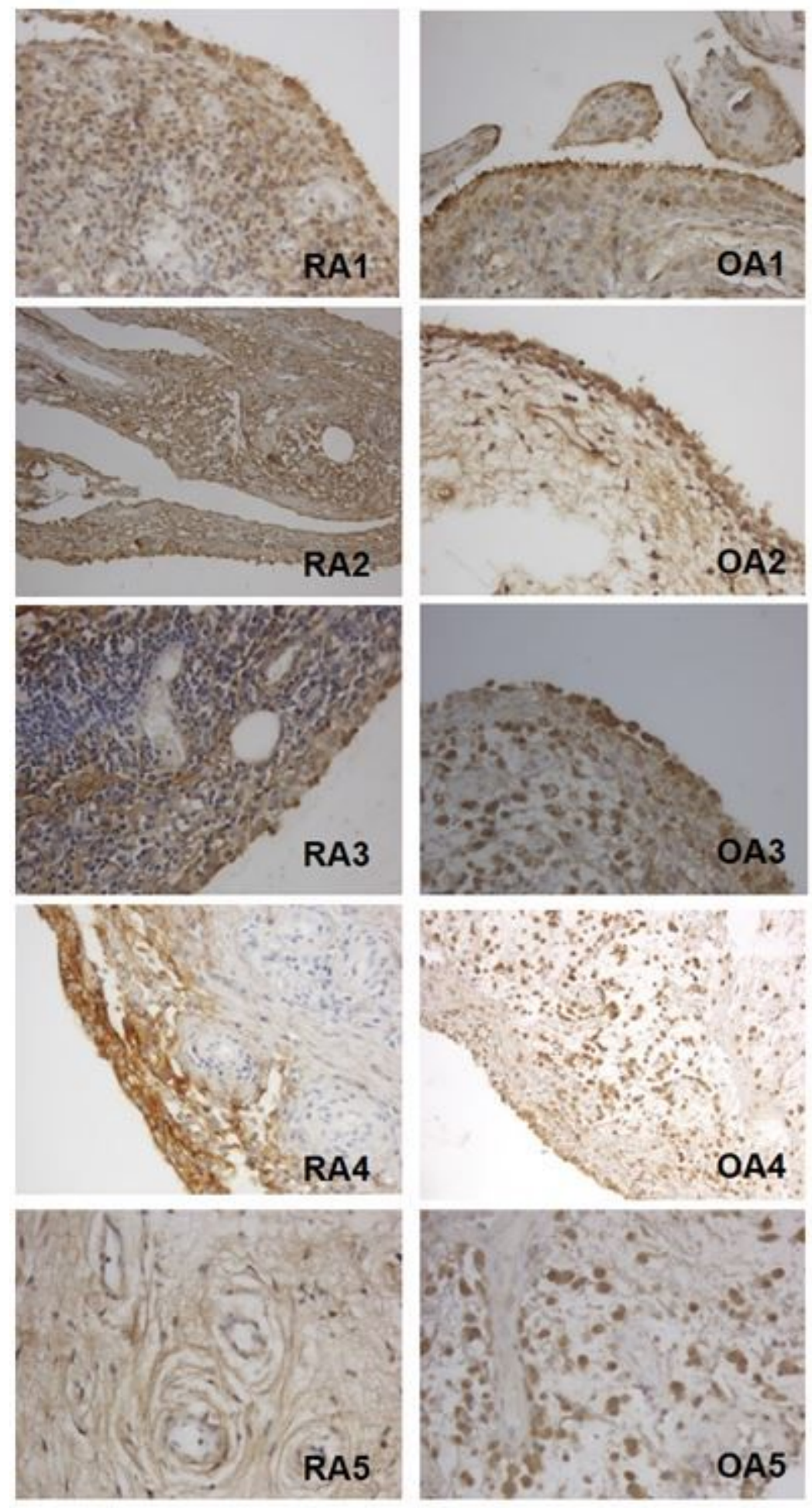

Figure 2

The comparison of syndecan-4 expression in synovial tissue between 5 RA patients and 5 OA patients. RA: rheumatoid arthritis; OA: osteoarthritis. 

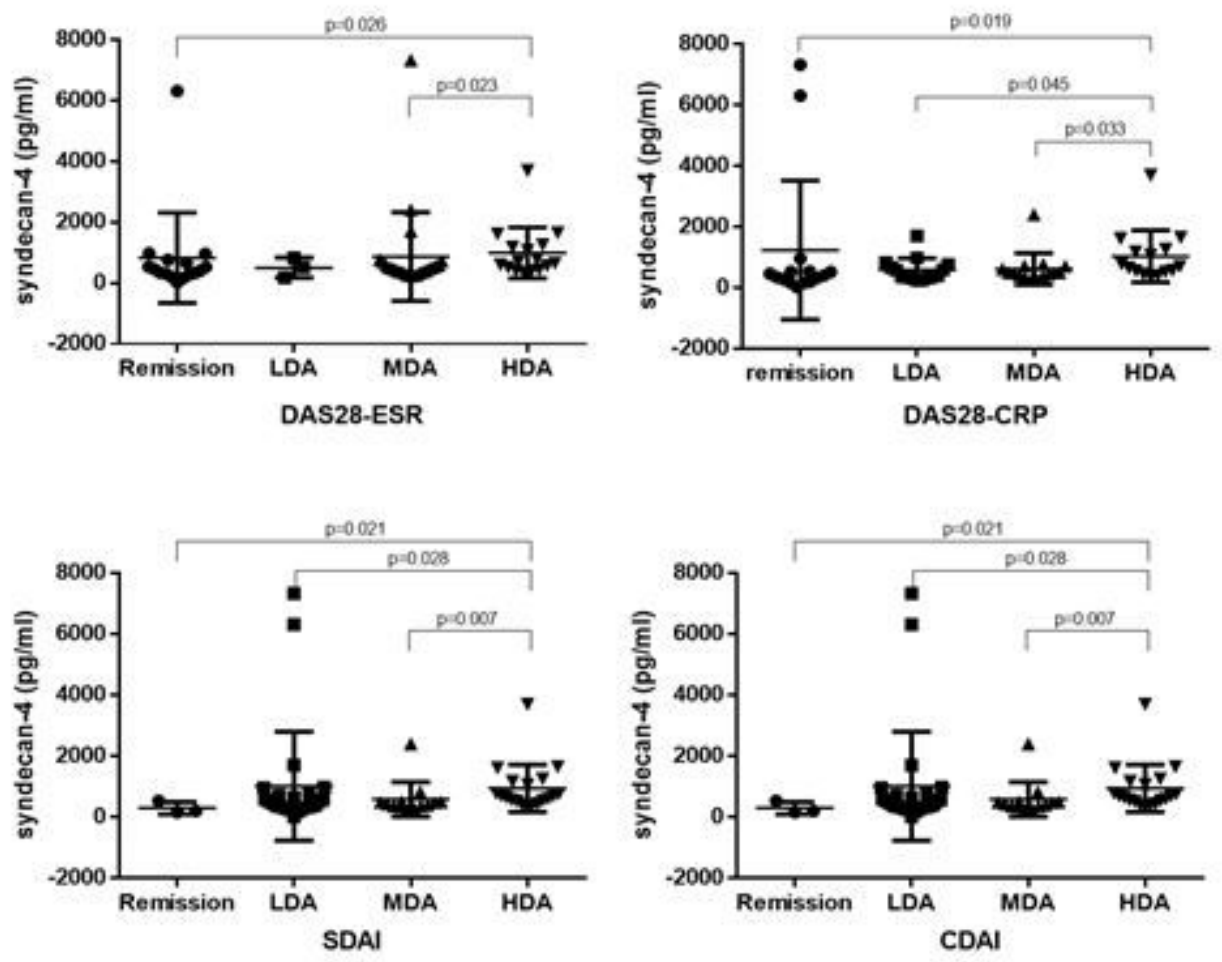

Figure 3

The comparison of serum syndecn-4 concentration among different disease activity groups based on DAS28-ESR, DAS28-CRP, SDAI and CDAI in 60 RA patients. DAS: disease activity; ESR: erythrocyte sedimentation rate; CRP: C-reactive protein; SDAl: simplified disease activity index; CDAl: clinical disease activity index; LDA: low disease activity; MDA: moderate disease activity; HDA: high disease activity.

\section{Supplementary Files}

This is a list of supplementary files associated with this preprint. Click to download.

- SI.docx 